\title{
Amyloid Imaging
}

Jessica Williams, CNMT, RT(N), FSNMMI-TS

\section{Rationale}

Amyloid imaging is performed to assist in the detection of $\beta$-amyloid plaques. Although these plaques are believed to be one of the causes of cognitive impairment (CI), a positive scan does not establish a diagnosis of Alzheimer disease or other cognitive disorder.

\section{Clinical Indications}

- Assessment of progressive or persistent unexplained mild CI.

- Evaluation of patients with known, progressive dementia and atypically early age of onset.

- Evaluation of patients fitting the core clinical criteria for possible diagnosis of Alzheimer disease, but the clinical presentation is either atypical or etiologically mixed.

\section{Contraindications/Inappropriate Use}

Pregnancy and breastfeeding are contraindicated but are not absolute contraindications as there are no known effects. Instructions should be provided to the patient for breast feeding and should follow the institution's policies. This scan should only be performed on pregnant women if there is a clear clinical benefit.

According to Society of Nuclear Medicine and Molecular Imaging standards and European Association of Nuclear Medicine practice guidelines, there are times when use of amyloid imaging can be considered inappropriate.

- Evaluation of dementia severity.

- Assessment of asymptomatic individuals with family history.

- Assessment of asymptomatic individuals who carry APOE- $\varepsilon 4$ genotype.

- Evaluation of asymptomatic individuals.

- Nonmedical use such as employment screening and legal.

- Evaluation of individuals with cognitive complaint that has not been confirmed.

- Assessment of individuals with core criteria for Alzheimer disease with typical age of onset.
- In lieu of genotyping for suspected autosomal dominant mutation carriers.

\section{Patient Preparation/Education}

- If the patient is of childbearing age, a pregnancy test should be performed.

- The patient may eat and take any necessary medications.

- History should be obtained to include symptoms, current medications, relevant personal history, and recent imaging procedures.

Patient education should include careful explanation of the procedure. It may be necessary to explain the procedure multiple times or in different ways as the patient may have severe CI. The patient may need constant reminders of the need for his or her cooperation. It is often helpful to have a family member or guardian accompany the patient. Some patients may not be able to cooperate with instructions and may need sedation. In that case, institutional guidelines should be followed.

\section{Protocol/Acquisition Instructions}

There are currently 3 Food and Drug Administrationapproved radiopharmaceuticals available in the Unites States: ${ }^{18} \mathrm{~F}$-florbetapir, ${ }^{18} \mathrm{~F}$-flutemetamol, and ${ }^{18} \mathrm{~F}$-florbetaben. Doses, uptake times, and acquisition times will vary dependent on which radiopharmaceutical is used.

The patient is interviewed, an intravenous catheter is placed, and the patient is injected with a bolus injection via the catheter with 1 of the 3 radiopharmaceuticals. The injection is immediately followed by $5-15 \mathrm{~mL}$ of $0.9 \%$ sterile sodium chloride to ensure dose delivery. Once the radiopharmaceutical is injected, the catheter can be removed, and the patient should relax during the uptake time.

After waiting the allotted uptake time, the patient should be instructed to empty his or her bladder, for comfort while imaging takes place. The patient should be positioned supine on the imaging couch with the head in a head holder. The whole of the brain should be in the field of view. If possible, extreme extension or flexion of the neck

\begin{tabular}{lcc}
\hline Radiopharmaceutical & Recommended dose & Uptake time \\
\hline 18 F-florbetapir & $10 \mathrm{mCi}(370 \mathrm{MBq})$ & $30-50 \mathrm{~min}$ \\
18 F-flutemetamol & $5 \mathrm{mCi} \mathrm{(185} \mathrm{MBq})$ & $60-120 \mathrm{~min}$ \\
18 F-florbetaben & $8.1 \mathrm{mCi}(300 \mathrm{MBq})$ & $10-20 \mathrm{~min}$ \\
\hline
\end{tabular}




\begin{tabular}{|c|c|}
\hline \multicolumn{2}{|c|}{ Standard Acquisition Protocol } \\
\hline Camera type & PET or PET/CT \\
\hline Energy peak & 511 \\
\hline Attenuation correction & $\begin{array}{l}\text { PET: cesium or germanium } \\
\text { sources, PET/CT: CT acquisition }\end{array}$ \\
\hline Patient position & Supine, arms down \\
\hline Acquisition type & 3-dimensional \\
\hline Time/bed position & See radiopharmaceutical \\
\hline Bed position & 1 \\
\hline
\end{tabular}

should be avoided. The patient should be made as comfortable as possible with the head secured by flexible head restraints.

Images should be acquired in 3-dimensional data acquisition mode with the appropriate data corrections.

\section{Image Processing}

- Image reconstruction should incorporate attenuation correction with the transaxial pixel sizes between 2 and $3 \mathrm{~mm}$ and slice thickness between 2 and $4 \mathrm{~mm}$.

- A minimum of 16-bit pixels for PET display is necessary to provide an adequate value range.

- PET images should be displayed in the transaxial orientation using gray scale or inverse gray scale. Color

\begin{tabular}{cc}
\hline Radiopharmaceutical & Display parameter \\
\hline${ }^{18} \mathrm{~F}$-florbetapir & $\begin{array}{c}\text { Maximum intensity of the display } \\
\text { scale should be set to the } \\
\text { brightest region of overall } \\
\text { brain uptake }\end{array}$ \\
& Scale intensity should be set to \\
& $90 \%$ in the pons region \\
${ }^{18} \mathrm{~F}$-flutemetamol & The white matter maximum \\
& should be the reference \\
\hline${ }^{18} \mathrm{~F}$-florbetaben & \\
\end{tabular}

scale may be used and is dependent on the recommendation of the manufacturer.

- Image size should be optimized to evaluate the graywhite matter.

\section{REFERENCES}

Farrell MB, Mantel ES, Basso DA, et al. The central nervous system: PET-amyloid brain imaging. In: Farrell MB, ed. Quick Reference Protocol Manual for Nuclear Medicine Technologist. Reston, VA: Society of Nuclear Medicine and Molecular Imaging; 2014:133-134.

Florbetapir F18 [package insert]. Indianapolis, IN: Eli Lily and Company; 2013. Flutametamol F18 [package insert]. Arlington Heights, IL: GE Healthcare; 2017.

Florbetaben F18 [package insert]. Dulles, VA: Piramal Imaging; 2014.

Minoshima S, Drzezga AE, Barthel H, et al. SNMMI procedure standard/EANM practice guideline for amyloid PET imaging of the brain 1.0. J Nucl Med. 2016;57:1316-1322. 